\title{
The Bochner Technique and Weighted Curvatures
}

Peter PETERSEN and Matthias WINK

Department of Mathematics, University of California, 520 Portola Plaza, Los Angeles, CA, 90095, USA

E-mail: petersen@math.ucla.edu,wink@math.ucla.edu

Received May 22, 2020, in final form June 29, 2020; Published online July 09, 2020

https://doi.org/10.3842/SIGMA.2020.064

Abstract. In this note we study the Bochner formula on smooth metric measure spaces. We introduce weighted curvature conditions that imply vanishing of all Betti numbers.

Key words: Bochner technique; smooth metric measure spaces; Hodge theory

2020 Mathematics Subject Classification: 53B20; 53C20; 53C21; 53C23; 58A14

\section{Introduction}

Let $(M, g)$ be an oriented Riemannian manifold, let vol $_{g}$ denote its volume form and let $f$ be a smooth function on $M$. The triple $\left(M, g, e^{-f} \operatorname{vol}_{g}\right)$ is called a smooth metric measure space. Based on considerations from diffusion processes, Bakry-Émery [1] introduced the tensor

$$
\operatorname{Ric}_{f}=\operatorname{Ric}+\operatorname{Hess} f
$$

as a weighted Ricci curvature for a geometric measure space. In fact, this tensor appeared earlier in work of Lichnerowicz [3]. Volume comparison theorems for smooth metric measure spaces with Ric $_{f}$ bounded from below have been established by Qian [7], Lott [4], Bakry-Qian [2] and Wei-Wylie [8].

In this note we study the Bochner technique on smooth metric measure spaces. The distortion of the volume element introduces a diffusion term to the Bochner formula

$$
\Delta_{f} \omega=\left(d d_{f}^{*}+d_{f}^{*} d\right) \omega=\nabla_{f}^{*} \nabla \omega+\operatorname{Ric}(\omega)-(\operatorname{Hess} f) \omega,
$$

where Ric is the Bochner operator on $p$-forms. Lott [4] proved that if $\operatorname{Ric}_{f} \geq 0$, then all $\Delta_{f}$-harmonic 1-forms are parallel and, for compact manifolds, $H^{1}(M ; \mathbb{R})$ is isomorphic to the space of all parallel 1-forms $\omega$ which satisfy $\left\langle\nabla e^{-f}, \omega\right\rangle=0$. Moreover, if $\operatorname{Ric}_{f}>0$, then all $\Delta_{f}$-harmonic 1-forms vanish.

We introduce new weighted curvature conditions that imply rigidity and vanishing results for $\Delta_{f}$-harmonic $p$-forms for $p \geq 1$. We can restrict to $p$-forms $\omega$ for $1 \leq p \leq\left\lfloor\frac{n}{2}\right\rfloor$ since $\omega$ is parallel if and only if $* \omega$ is parallel, where $*$ denotes the Hodge star.

By convention, we will refer to the eigenvalues of the curvature operator simply as the eigenvalues of the associated curvature tensor.

Theorem. Let $\left(M^{n}, g, e^{-f} \operatorname{vol}_{g}\right)$ be a smooth metric measure space. For $1 \leq p<\frac{n}{2}$ set

$$
h=\frac{1}{n-2 p} \operatorname{Hess} f-\frac{\Delta f}{2(n-p)(n-2 p)} g .
$$

Let $\omega$ be a $\Delta_{f}$-harmonic p-form with $|\omega| \in L^{2}\left(M, e^{-f} \operatorname{vol}_{g}\right)$ for $1 \leq p<\frac{n}{2}$. Let $\lambda_{1} \leq \cdots \leq \lambda_{\left(\begin{array}{c}n \\ 2\end{array}\right)}$ denote the eigenvalues of the weighted curvature tensor $\mathrm{Rm}+h \otimes g$.

This paper is a contribution to the Special Issue on Scalar and Ricci Curvature in honor of Misha Gromov on his 75th Birthday. The full collection is available at https://www.emis.de/journals/SIGMA/Gromov.html 
If $\lambda_{1}+\cdots+\lambda_{n-p} \geq 0$, then $\omega$ is parallel. If in addition $M$ is compact, then $H^{p}(M)=\{\omega \in$ $\Omega^{p}(M) \mid \nabla \omega=0$ and $\left.i_{\nabla f} \omega=0\right\}$.

If $\lambda_{1}+\cdots+\lambda_{n-p}>0$, then $\omega$ vanishes. If in addition $M$ is compact, then the Betti numbers $b_{p}(M)$ and $b_{n-p}(M)$ vanish for $1 \leq p<\frac{n}{2}$.

For $p=1$ the Ricci curvature of the modified curvature tensor is the Bakry-Émery Ricci tensor, and the assumption in the Theorem implies that it is nonnegative. In this sense the Theorem is a generalization of Lott's [4] results for 1-forms.

A stronger curvature assumption also allows control in the middle dimension $p=\frac{n}{2}$. Recall that a curvature tensor is $l$-nonnegative (positive) if the sum of its lowest $l$ eigenvalues is nonnegative (positive).

Proposition. Let $\left(M^{n}, g, e^{-f} \operatorname{vol}_{g}\right)$ be a smooth metric measure space. Let $\mu_{1} \leq \cdots \leq \mu_{n}$ denote the eigenvalues of Hess $f$ and let $1 \leq p \leq\left\lfloor\frac{n}{2}\right\rfloor$.

Let $\omega$ be a $\Delta_{f}$-harmonic p-form with $|\omega| \in L^{2}\left(M, e^{-f} \operatorname{vol}_{g}\right)$. If the weighted curvature tensor

$$
\mathrm{Rm}+\frac{\sum_{i=1}^{p} \mu_{i}}{2 p(n-p)} g \oslash g
$$

is $(n-p)$-nonnegative, then $\omega$ is parallel. If it is $(n-p)$-positive, then $\omega$ vanishes.

In particular, if $M$ is compact, then $H^{p}(M)=\left\{\omega \in \Omega^{p}(M) \mid \nabla \omega=0\right.$ and $\left.i_{\nabla f} \omega=0\right\}$ and in case the weighted curvature tensor is $(n-p)$-positive, the Betti numbers $b_{p}(M)$ and $b_{n-p}(M)$ vanish.

The notation in this paper builds up on the presentation in [5, Chapter 9] and [6].

\section{Preliminaries}

\subsection{Algebraic curvature tensors}

For an $n$-dimensional Euclidean vector space $(V, g)$ let $\mathcal{T}^{(0, k)}(V)$ denote the vector space of $(0, k)$-tensors and $\operatorname{Sym}^{2}(V)$ the vector space of symmetric $(0,2)$-tensors on $V$.

Let $\mathcal{C}(V)$ denote the vector space of $(0,4)$-tensors with $T(X, Y, Z, W)=-T(Y, X, Z, W)=$ $T(Z, W, X, Y)$. If $T$ also satisfies the algebraic Bianchi identity, then $T$ is called algebraic curvature tensor, $T \in \mathcal{C}_{B}(V)$.

The Kulkarni-Nomizu product of $S_{1}, S_{2} \in \operatorname{Sym}^{2}(V)$ is given by

$$
\begin{aligned}
\left(S_{1} \oplus S_{2}\right)(X, Y, Z, W)= & S_{1}(X, Z) S_{2}(Y, W)-S_{1}(X, W) S_{2}(Y, Z) \\
& +S_{1}(Y, W) S_{2}(X, Z)-S_{1}(Y, Z) S_{2}(X, W) .
\end{aligned}
$$

With this convention the algebraic curvature tensor $I=\frac{1}{2} g \otimes g$ corresponds to the curvature tensor of the unit sphere.

Recall that the decomposition of $\mathcal{C}(V)$ into $O(n)$-irreducible components is given by

$$
\mathcal{C}(V)=\langle I\rangle \oplus\langle\text { Ric }\rangle \oplus\langle W\rangle \oplus \Lambda^{4} V
$$

where $\langle$ Ric $\rangle=S_{0}^{2}(V) \otimes g$ is the subspace of algebraic curvature tensors of trace-free Ricci type, $S_{0}^{2}(V)=\left\{h \in \operatorname{Sym}^{2}(V) \mid \operatorname{tr}(h)=0\right\}$, and $\langle W\rangle$ denotes the subspace of Weyl tensors.

Explicitly, every algebraic curvature tensor decomposes as

$$
\mathrm{Rm}=\frac{\text { scal }}{2(n-1) n} g \oplus g+\frac{1}{n-2} \operatorname{Ric} \oplus g+W .
$$




\subsection{Lichnerowicz Laplacians on smooth metric measure spaces}

Let $(M, g, f)$ be a smooth metric measure space. The formal adjoints of the exterior and covariant derivative with respect to the measure $e^{-f} \operatorname{vol}_{g}$ are given by

$$
d_{f}^{*}=d^{*}+i_{\nabla f} \quad \text { and } \quad \nabla_{f}^{*}=\nabla^{*}+i_{\nabla f} .
$$

More generally, for a vector field $U$ on $M$, we will consider

$$
d_{U}^{*}=d^{*}+i_{U} \quad \text { and } \quad \nabla_{U}^{*}=\nabla^{*}+i_{U} .
$$

The associated generalized Lichnerowicz Laplacian on $(0, k)$-tensors is given by

$$
\Delta_{U} T=\nabla_{U}^{*} \nabla T+\operatorname{Ric}(T)-(\nabla U) T
$$

where the curvature term is given by

$$
\operatorname{Ric}(T)\left(X_{1}, \ldots, X_{k}\right)=\sum_{i=1}^{k} \sum_{j=1}^{n}\left(R\left(X_{i}, e_{j}\right) T\right)\left(X_{1}, \ldots, e_{j}, \ldots, X_{k}\right) .
$$

A tensor $T$ is called $U$-harmonic if $\Delta_{U} T=0$.

To emphasize that the curvature term is calculated with respect to the curvature tensor $\mathrm{Rm}$, we will also write $\operatorname{Ric}_{\operatorname{Rm}}(T)$ for $\operatorname{Ric}(T)$.

Recall that for an endomorphism $L$ of $V$ and a $(0, k)$-tensor $T$ we have

$$
(L T)\left(X_{1}, \ldots, X_{k}\right)=-\sum_{i=1}^{k} T\left(X_{1}, \ldots, L\left(X_{i}\right), \ldots, X_{k}\right) .
$$

In particular, the Ricci identity implies that the definition of the curvature term in the Lichnerowicz Laplacian naturally carries over to algebraic curvature tensors.

Proposition 2.1. Let $(M, g)$ be a Riemannian manifold and $U$ a vector field on $M$. For $a(0, k)$-tensor $T$ on $M$ set $\operatorname{Ric}_{U}(T)=\operatorname{Ric}(T)-(\nabla U) T$.

(a) Every p-form satisfies

$$
\left(d d_{U}^{*}+d_{U}^{*} d\right) \omega=\nabla_{U}^{*} \nabla \omega+\operatorname{Ric}_{U}(\omega) .
$$

(b) Every symmetric (0,2)-tensor satisfies

$$
\left(\nabla_{X} \nabla_{U}^{*} T\right)(X)+\left(\nabla_{U}^{*} d^{\nabla} T\right)(X, X)=\left(\nabla_{U}^{*} \nabla T\right)(X, X)+\frac{1}{2}\left(\operatorname{Ric}_{U} T\right)(X, X),
$$

where $d^{\nabla} T(Z, X, Y)=\left(\nabla_{X} T\right)(Y, Z)-\left(\nabla_{Y} T\right)(X, Z)$.

Proof. (a) The case $U=0$ recovers the well-known Bochner formula. The generalized Hodge Laplacian satisfies

$$
d d_{U}^{*}+d_{U}^{*} d=d d^{*}+d^{*} d+d i_{U}+i_{U} d=\Delta+L_{U} .
$$

In addition to the classical Lichnerowicz Laplacian we have on the right hand side

$$
\nabla_{U}-(\nabla U)=L_{U}
$$

and thus all diffusion terms balance out. 
(b) As in (a), it suffices to consider all terms that depend on $U$ and show that

$$
\left(\nabla_{X} i_{U} h\right)(X)+\left(i_{U} d^{\nabla} h\right)(X, X)=\left(\nabla_{U} h\right)(X, X)-\frac{1}{2}((\nabla U) h)(X, X) .
$$

This is a straightforward calculation

$$
\begin{aligned}
& \left(\nabla_{X} i_{U} h\right)(X)+\left(i_{U} d^{\nabla} h\right)(X, X) \\
& \quad=\left(\nabla_{X} h\right)(U, X)+h\left(\nabla_{X} U, X\right)+\left(\nabla_{U} h\right)(X, X)-\left(\nabla_{X} h\right)(U, X) \\
& \quad=\left(\nabla_{U} h\right)(X, X)+h\left(\nabla_{X} U, X\right) \\
& \quad=\left(\nabla_{U} h\right)(X, X)-\frac{1}{2}((\nabla U) h)(X, X) .
\end{aligned}
$$

Remark 2.2. The curvature tensor Rm of a Riemannian manifold satisfies

$$
\begin{aligned}
\nabla_{U}^{*} \nabla \mathrm{Rm}+\frac{1}{2} \operatorname{Ric}_{U}(\mathrm{Rm})= & \frac{1}{2}\left(\nabla_{X} \nabla_{U}^{*} \operatorname{Rm}\right)(Y, Z, W)-\frac{1}{2}\left(\nabla_{Y} \nabla_{U}^{*} \operatorname{Rm}\right)(X, Z, W) \\
& +\frac{1}{2}\left(\nabla_{Z} \nabla_{U}^{*} \operatorname{Rm}\right)(W, X, Y)-\frac{1}{2}\left(\nabla_{W} \nabla_{U}^{*} \operatorname{Rm}\right)(Z, X, Y) .
\end{aligned}
$$

A straightforward computation based on the second Bianchi identity shows that all terms that involve $U$ cancel.

The Bochner technique with diffusion relies on the following basic observations. Firstly, the maximum principle implies:

Lemma 2.3. Let $(M, g)$ be a Riemannian manifold, $U$ a vector field on $M$. Let $T$ be a tensor such that

$$
g\left(\nabla_{U}^{*} \nabla T, T\right) \leq 0 .
$$

If $|T|$ has a maximum, then $T$ is parallel.

Remark 2.4. Note that a $p$-form $\omega$ satisfies $\left(d d_{U}^{*}+d_{U}^{*} d\right) \omega=0$ if and only if $d \omega=0$ and $d_{U}^{*} \omega=0$.

As in [4], if $M$ is compact and oriented, standard elliptic theory implies that

$$
H^{p}(M)=\left\{\omega \in \Omega^{p}(M) \mid d \omega=0 \text { and } d_{U}^{*} \omega=0\right\} .
$$

Suppose that $\operatorname{Ric}_{U} \geq 0$ on $p$-forms. It follows that a $p$-form $\omega$ is $U$-harmonic if and only if $\omega$ is parallel and $i_{U} \omega=0$. Thus,

$$
H^{p}(M)=\left\{\omega \in \Omega^{p}(M) \mid \nabla \omega=0 \text { and } i_{U} \omega=0\right\} .
$$

If $U=\nabla f$, then we can use integration to conclude:

Lemma 2.5. Let $(M, g, f)$ be a smooth metric measure space with $\int_{M} e^{-f} \operatorname{vol}_{g}<\infty$. If $T$ is a $(0, k)$-tensor with $|T| \in L^{2}\left(M, e^{-f} \operatorname{vol}_{g}\right)$ and

$$
g\left(\nabla_{f}^{*} \nabla T, T\right) \leq 0,
$$

then $T$ is parallel. 


\section{Weighted Lichnerowicz Laplacians}

The idea of this section is to define a weighted curvature tensor $\widetilde{\mathrm{Rm}}$ so that for a given symmetric tensor $S$ the curvature term of the Lichnerowicz Laplacian satisfies

$$
g\left(\operatorname{Ric}_{\mathrm{Rm}}(T)-(S) T, T\right)=g\left(\operatorname{Ric}_{\widetilde{\operatorname{Rm}}}(T), T\right) .
$$

This will be achieved by adding a weight to the Ricci tensor of Rm, leaving the Weyl curvature unchanged. The specific weight will depend on the irreducible components of the tensors of type $T$, e.g., it is different for forms and symmetric tensors.

Let $T$ be a $(0, k)$-tensor. For $\tau_{i j} \in S_{k}$ let $T \circ \tau_{i j}$ denote the transposition of the $i$-th and $j$-th entries of $T$ and for $h \in \operatorname{Sym}^{2}(V)$ let $c_{i j}(h \otimes T)$ denote the contraction of $h$ with the $i$-th and $j$-th entries of $T$.

Proposition 3.1. For $h \in \operatorname{Sym}^{2}(V)$ let $H: V \rightarrow V$ denote the associated symmetric operator. If $T \in \mathcal{T}^{(0, k)}(V)$, then

$$
\begin{aligned}
\operatorname{Ric}_{h \otimes g}(T)\left(X_{1}, \ldots, X_{k}\right)= & 2 \sum_{i \neq j}\left(T \circ \tau_{i j}\right)\left(X_{1}, \ldots, H\left(X_{i}\right), \ldots, X_{k}\right) \\
& -\sum_{i \neq j} g\left(X_{i}, X_{j}\right) c_{i j}(h \otimes T)\left(X_{1}, \ldots, \widehat{X}_{i}, \ldots, \widehat{X}_{j}, \ldots, X_{k}\right) \\
& -\sum_{i \neq j} h\left(X_{i}, X_{j}\right) c_{i j}(g \otimes T)\left(X_{1}, \ldots, \widehat{X}_{i}, \ldots, \widehat{X}_{j}, \ldots, X_{k}\right) \\
& -(n-2)(H T)\left(X_{1}, \ldots, X_{k}\right)+k \cdot \operatorname{tr}(h) T\left(X_{1}, \ldots, X_{k}\right) .
\end{aligned}
$$

Proof. The algebraic curvature tensor $R=h \otimes g$ satisfies

$$
\begin{aligned}
R(X, Y, Z, W)= & g(H(X), Z) g(Y, W)-g(Y, Z) g(H(X), W) \\
& +g(X, Z) g(H(Y), W)-g(H(Y), Z) g(X, W)
\end{aligned}
$$

and hence

$$
R(X, Y) Z=(H(X) \wedge Y+X \wedge H(Y)) Z
$$

is the corresponding $(1,3)$-tensor. It follows that

$$
\begin{aligned}
\operatorname{Ric}_{h \oplus g}(T)\left(X_{1}, \ldots, X_{k}\right)= & \sum_{i=1}^{k} \sum_{a=1}^{n}\left(R\left(X_{i}, e_{a}\right) T\right)\left(X_{1}, \ldots, e_{a}, \ldots, X_{k}\right) \\
= & \sum_{i=1}^{k} \sum_{a=1}^{n}\left(\left(H\left(X_{i}\right) \wedge e_{a}\right) T\right)\left(X_{1}, \ldots, e_{a}, \ldots, X_{k}\right) \\
& +\sum_{i=1}^{k} \sum_{a=1}^{n}\left(\left(X_{i} \wedge H\left(e_{a}\right)\right) T\right)\left(X_{1}, \ldots, e_{a}, \ldots, X_{k}\right) .
\end{aligned}
$$

It is straightforward to calculate

$$
\begin{aligned}
& \sum_{i=1}^{k} \sum_{a=1}^{n}\left(\left(X_{i} \wedge H\left(e_{a}\right)\right) T\right)\left(X_{1}, \ldots, e_{a}, \ldots, X_{k}\right) \\
& \quad=\sum_{i \neq j} \sum_{a=1}^{n} T\left(X_{1}, \ldots,\left(H\left(e_{a}\right) \wedge X_{i}\right) X_{j}, \ldots, e_{a}, \ldots, X_{k}\right)
\end{aligned}
$$




$$
\begin{aligned}
& +\sum_{i=1}^{k} \sum_{a=1}^{n} T\left(X_{1}, \ldots,\left(H\left(e_{a}\right) \wedge X_{i}\right) e_{a}, \ldots, X_{k}\right) \\
& =\sum_{i \neq j} \sum_{a=1}^{n} T\left(X_{1}, \ldots, g\left(H\left(e_{a}\right), X_{j}\right) X_{i}-g\left(X_{i}, X_{j}\right) H\left(e_{a}\right), \ldots, e_{a}, \ldots, X_{k}\right) \\
& +\sum_{i=1}^{k} \sum_{a=1}^{n} T\left(X_{1}, \ldots, g\left(H\left(e_{a}\right), e_{a}\right) X_{i}-g\left(e_{a}, X_{i}\right) H\left(e_{a}\right), \ldots, X_{k}\right) \\
& =\sum_{i \neq j} \sum_{a=1}^{n} T\left(X_{1}, \ldots, g\left(e_{a}, H\left(X_{j}\right)\right) X_{i}, \ldots, e_{a}, \ldots, X_{k}\right) \\
& -\sum_{i \neq j} \sum_{a=1}^{n} g\left(X_{i}, X_{j}\right) T\left(X_{1}, \ldots, H\left(e_{a}\right), \ldots, e_{a}, \ldots, X_{k}\right) \\
& +\sum_{i=1}^{k} \sum_{a=1}^{n} h\left(e_{a}, e_{a}\right) T\left(X_{1}, \ldots, X_{k}\right)-\sum_{i=1}^{k} \sum_{a=1}^{n} T\left(X_{1}, \ldots, H\left(g\left(e_{a}, X_{i}\right) e_{a}\right), \ldots, X_{k}\right) \\
& =\sum_{i \neq j} T\left(X_{1}, \ldots, X_{i}, \ldots, H\left(X_{j}\right), \ldots, X_{k}\right) \text { [here } X_{i} \text { is in the } \mathrm{j} \text {-th position] } \\
& -\sum_{i \neq j} \sum_{a, b=1}^{n} g\left(X_{i}, X_{j}\right) h\left(e_{a}, e_{b}\right) T\left(X_{1}, \ldots, e_{b}, \ldots, e_{a}, \ldots, X_{k}\right)+k \cdot \operatorname{tr}(h) T\left(X_{1}, \ldots, X_{k}\right) \\
& -\sum_{i=1}^{k} T\left(X_{1}, \ldots, H\left(X_{i}\right), \ldots, X_{k}\right) \\
& \left.=\sum_{i \neq j}\left(T \circ \tau_{i j}\right)\left(X_{1}, \ldots, H\left(X_{j}\right), \ldots, X_{i}, \ldots, X_{k}\right) \text { [here } H\left(X_{j}\right) \text { is in the } \mathrm{j} \text {-th position }\right] \\
& -\sum_{i \neq j} g\left(X_{i}, X_{j}\right) c_{i j}(h \otimes T)\left(X_{1}, \ldots, \widehat{X_{i}}, \ldots, \widehat{X_{j}}, \ldots, X_{k}\right) \\
& +k \cdot \operatorname{tr}(h) T\left(X_{1}, \ldots, X_{k}\right)+(H T)\left(X_{1}, \ldots, X_{k}\right) \text {. }
\end{aligned}
$$

Similarly one computes

$$
\begin{aligned}
\sum_{i=1}^{k} \sum_{a=1}^{n} & \left(\left(H\left(X_{i}\right) \wedge e_{a}\right) T\right)\left(X_{1}, \ldots, e_{a}, \ldots, X_{k}\right) \\
= & \sum_{i \neq j}\left(T \circ \tau_{i j}\right)\left(X_{1}, \ldots, X_{j}, \ldots, H\left(X_{i}\right), \ldots, X_{k}\right) \text { [here } X_{j} \text { is in the j-th position] } \\
& \quad-\sum_{i \neq j} h\left(X_{i}, X_{j}\right) c_{i j}(g \otimes T)\left(X_{1}, \ldots, \widehat{X_{i}}, \ldots, \widehat{X_{j}}, \ldots, X_{k}\right)-(n-1)(H T)\left(X_{1}, \ldots, X_{k}\right) .
\end{aligned}
$$

Adding up both terms yields $\operatorname{Ric}_{h \otimes g}(T)$ as claimed.

Proposition 3.2. Let $(V, g)$ be an $n$-dimensional Euclidean vector space and $h \in \operatorname{Sym}^{2}(V)$. The following hold:

1. Every $T \in \operatorname{Sym}^{2}(V)$ satisfies

$$
\begin{aligned}
& \operatorname{Ric}_{h \otimes g}(T)=-n H T-2\langle T, h\rangle g-2 \operatorname{tr}(T) h+2 \operatorname{tr}(h) T, \\
& g\left(\operatorname{Ric}_{h \otimes g}(T), T\right)=-n g(H T, T)-4 \operatorname{tr}(T)\langle T, h\rangle+2 \operatorname{tr}(h)|T|^{2} .
\end{aligned}
$$


2. Every p-form $\omega$ satisfies

$$
\begin{aligned}
& \operatorname{Ric}_{h \oplus g}(\omega)=-(n-2 p) H \omega+p \operatorname{tr}(h) \omega, \\
& g\left(\operatorname{Ric}_{h \oplus g}(\omega), \omega\right)=-(n-2 p) g(H \omega, \omega)+p \operatorname{tr}(h)|\omega|^{2} .
\end{aligned}
$$

3. Every algebraic $(0,4)$-curvature tensor $\mathrm{Rm}$ satisfies

$$
\operatorname{Ric}_{h \otimes g}(\mathrm{Rm})=-2(h \otimes \mathrm{Ric})-2 g \otimes\left(c_{24}(h \otimes \mathrm{Rm})\right)-(n-2) H \mathrm{Rm}+4 \operatorname{tr}(h) \mathrm{Rm} .
$$

Proof. (a) Due to the symmetry of $T$ it follows that

$$
\begin{aligned}
\operatorname{Ric}_{h \otimes g}(T)\left(X_{1}, X_{2}\right)= & 2\left\{T\left(H\left(X_{1}\right), X_{2}\right)+T\left(X_{1}, H\left(X_{2}\right)\right\}\right. \\
& -2\left\{g\left(X_{1}, X_{2}\right)\langle h, T\rangle+h\left(X_{1}, X_{2}\right) \operatorname{tr}(T)\right\} \\
& -(n-2)(H T)\left(X_{1}, X_{2}\right)+2 \operatorname{tr}(h) T\left(X_{1}, X_{2}\right) .
\end{aligned}
$$

(b) Since $\omega \circ \tau_{i j}=-\omega$ for every transposition $\tau_{i j}$ it follows that

$$
\begin{aligned}
\sum_{i \neq j}\left(\omega \circ \tau_{i j}\right)\left(X_{1}, \ldots, H\left(X_{i}\right), \ldots, X_{p}\right) & =-\sum_{i \neq j} \omega\left(X_{1}, \ldots, H\left(X_{i}\right), \ldots, X_{p}\right) \\
& =-(p-1) \sum_{i=1}^{p} \omega\left(X_{1}, \ldots, H\left(X_{i}\right), \ldots, X_{p}\right) \\
& =(p-1)(H \omega)\left(X_{1}, \ldots, X_{p}\right)
\end{aligned}
$$

and furthermore $c_{i j}(g \otimes \omega)=c_{i j}(h \otimes \omega)=0$ for all $i \neq j$. This implies the claim.

(c) The symmetries of the curvature tensor imply that

$$
\begin{aligned}
& \sum_{i \neq j}\left(\operatorname{Rm} \circ \tau_{i j}\right)\left(X_{1}, \ldots, H\left(X_{i}\right), \ldots, X_{4}\right) \\
& \quad=(H \mathrm{Rm})\left(X_{1}, X_{2}, X_{3}, X_{4}\right)+(H \mathrm{Rm})\left(X_{2}, X_{3}, X_{1}, X_{4}\right)+(H \mathrm{Rm})\left(X_{3}, X_{1}, X_{2}, X_{4}\right)=0
\end{aligned}
$$

due to the first Bianchi identity.

Computing with respect to an orthonormal eigenbasis of $H$ it follows that

$$
\begin{aligned}
\left(g(\cdot, \cdot) c_{12}(h \otimes \operatorname{Rm})\right)(X, Y, Z, W) & =0, \\
\left(g(\cdot, \cdot) c_{13}(h \otimes \mathrm{Rm})\right)(X, Y, Z, W) & =\sum_{a, b=1}^{n} g(X, Z) \operatorname{Rm}\left(g\left(H\left(e_{a}\right), e_{b}\right) e_{b}, Y, e_{a}, W\right) \\
& =\sum_{a=1}^{n} g(X, Z) \operatorname{Rm}\left(H\left(e_{a}\right), Y, e_{a}, W\right) \\
& =\sum_{a=1}^{n} g(Z, X) \operatorname{Rm}\left(e_{a}, Y, H\left(e_{a}\right), W\right) \\
& =\left(g(\cdot, \cdot) c_{31}(h \otimes \operatorname{Rm})\right)(X, Y, Z, W) .
\end{aligned}
$$

This implies

$$
\begin{aligned}
& \sum_{i \neq j}\left(g(\cdot, \cdot) c_{i j}(h \otimes \operatorname{Rm})\right)(X, Y, Z, W) \\
& \quad=2 \sum_{i=1}^{n}\left\{g(X, Z) \operatorname{Rm}\left(H\left(e_{i}\right), Y, e_{i}, W\right)+g(X, W) \operatorname{Rm}\left(H\left(e_{i}\right), Y, Z, e_{i}\right)\right.
\end{aligned}
$$




$$
\begin{aligned}
& \left.+g(Y, Z) \operatorname{Rm}\left(X, H\left(e_{i}\right), e_{i}, W\right)+g(Y, W) \operatorname{Rm}\left(X, H\left(e_{i}\right), Z, e_{i}\right)\right\} \\
= & 2 \sum_{i=1}^{n}\left\{g(X, Z) \operatorname{Rm}\left(Y, H\left(e_{i}\right), W, e_{i}\right)-g(X, W) \operatorname{Rm}\left(Y, H\left(e_{i}\right), Z, e_{i}\right)\right. \\
& \left.-g(Y, Z) \operatorname{Rm}\left(X, H\left(e_{i}\right), W, e_{i}\right)+g(Y, W) \operatorname{Rm}\left(X, H\left(e_{i}\right), Z, e_{i}\right)\right\} \\
= & 2\left(g \otimes\left[\sum_{i=1}^{n} \operatorname{Rm}\left(\cdot, H\left(e_{i}\right), \cdot, e_{i}\right)\right]\right)(X, Y, Z, W) \\
= & 2\left(g \otimes c_{24}(h \otimes \operatorname{Rm})\right)(X, Y, Z, W) .
\end{aligned}
$$

Similarly it follows that

$$
\sum_{i \neq j}\left(h(\cdot, \cdot) c_{i j}(g \otimes \mathrm{Rm})\right)=2\left(h \otimes c_{24}(g \otimes \mathrm{Rm})\right)=2(h \otimes \mathrm{Ric}) .
$$

This completes the proof.

Remark 3.3. For a Weyl tensor $W$ and $h$ a symmetric $(0,2)$-tensor it is not hard to check that $\operatorname{Ric}_{h \otimes g}(W)$ satisfies

$$
\begin{aligned}
& g\left(\operatorname{Ric}_{h \otimes g}(W), W\right)=-(n-2) g(H W, W)+4 \operatorname{tr}(h)|W|^{2}, \\
& g\left(\operatorname{Ric}_{h \otimes g}(W), g \oplus \operatorname{Ric}\right)=-8(n-2)\left\langle c_{24}(h \otimes W), \operatorname{Ric}\right\rangle=-8(n-2)\left\langle c_{24}(h \otimes W), \operatorname{Ric}\right\rangle, \\
& g\left(\operatorname{Ric}_{h \otimes g}(W), g \otimes g\right)=0 .
\end{aligned}
$$

It is worth noting that there are trace-free symmetric $(0,2)$-tensors $h_{1}, h_{2}$ such that the curvature tensor $h_{1} \otimes h_{2}$ is Weyl.

The main Theorem follows as in Proposition 3.4 below by using Lemma 2.5 instead of Lemma 2.3. The description of the de Rham cohomology groups follows from Remark 2.4.

Proposition 3.4. Let $(M, g)$ be a Riemannian manifold and let $U$ be a vector field on $M$. Set $S=\nabla U$ and for $1 \leq p<\frac{n}{2}$ set

$$
H=\frac{1}{n-2 p} S-\frac{1}{2(n-p)(n-2 p)} \operatorname{tr}(S) I,
$$

where $I: T M \rightarrow T M$ denotes the identity operator.

Suppose that the eigenvalues $\lambda_{1} \leq \cdots \leq \lambda_{\left(\begin{array}{c}n \\ 2\end{array}\right)}$ of the weighted curvature tensor $\mathrm{Rm}+h \otimes g$ satisfy

$$
\lambda_{1}+\cdots+\lambda_{n-p} \geq 0
$$

and let $\omega$ be a $U$-harmonic $p$-form for $1 \leq p<\frac{n}{2}$.

If $|\omega|$ achieves a maximum, then $\omega$ is parallel. If in addition the inequality is strict, then $\omega$ vanishes.

Proof. Proposition 3.2 (b) and $-I \omega=p \omega$ imply that

$$
\begin{aligned}
g\left(\operatorname{Ric}_{h \otimes g} \omega, \omega\right) & =-(n-2 p) g(H \omega, \omega)+p \operatorname{tr}(h)|\omega|^{2}=-g(((n-2 p) H+\operatorname{tr}(h) I) \omega, \omega) \\
& =-g\left(\left(S-\frac{\operatorname{tr}(S)}{2(n-p)} I+\frac{\operatorname{tr}(S)}{2(n-p)} I\right) \omega, \omega\right)=-g(S \omega, \omega) .
\end{aligned}
$$

Thus the Bochner formula takes the form

$$
\Delta_{U} \omega=\nabla_{U}^{*} \nabla \omega+\operatorname{Ric}(\omega)-(\nabla U) \omega=\nabla_{U}^{*} \nabla \omega+\operatorname{Ric}_{\mathrm{Rm}+h \otimes g}(\omega) .
$$


The argument in $\left[6\right.$, proof of Theorem A] shows that $\operatorname{Ric}_{\mathrm{Rm}+h \otimes g}(\omega) \geq 0$. Lemma 2.3 implies the claim.

If the inequality is strict, then the same argument shows that $\operatorname{Ric}_{\mathrm{Rm}+h \otimes g}(\omega)>0$ unless $\omega=0$.

The above approach only works for $p=\frac{n}{2}$ if $S$ is a multiple of the identity. However, we have

Proposition 3.5. Let $(M, g)$ be an $n$-dimensional Riemannian manifold and let $U$ be a vector field on $M$. Set $S=\nabla U$ and fix $1 \leq p \leq\left\lfloor\frac{n}{2}\right\rfloor$. Let $\mu_{1} \leq \cdots \leq \mu_{n}$ denote the eigenvalues of $S$. Suppose that the weighted curvature tensor

$$
\mathrm{Rm}+\frac{\sum_{i=1}^{p} \mu_{i}}{2 p(n-p)} g \oplus g
$$

is $(n-p)$-nonnegative. If $\omega$ is a $U$-harmonic $p$-form $\omega$ such that $|\omega|$ has a maximum, then $\omega$ is parallel. If in addition the weighted curvature tensor is $(n-p)$-positive, then $\omega$ vanishes.

Proof. Calculating with respect to an orthonormal eigenbasis for $S$ it follows that

$$
-g((S \omega), \omega)=-\sum_{i_{1}<\cdots<i_{p}}(S \omega)_{i_{1} \ldots i_{p}} \omega_{i_{1} \ldots i_{p}}=\sum_{i_{1}<\cdots<i_{p}}\left(\sum_{j=1}^{p} \mu_{i_{j}}\right)\left(\omega_{i_{1} \ldots i_{p}}\right)^{2} \geq\left(\sum_{i=1}^{p} \mu_{i}\right)|\omega|^{2} .
$$

Let $\left\{\lambda_{\alpha}\right\}$ denote the eigenvalues of (the curvature operator associated to) $\mathrm{Rm}$ and let $\left\{\Xi_{\alpha}\right\}$ be an orthonormal eigenbasis. It follows from [6, Proposition 1.6] that

$$
g\left(\operatorname{Ric}_{\operatorname{Rm}}(\omega), \omega\right)-g(S \omega, \omega) \geq \sum_{\alpha} \lambda_{\alpha}\left|\Xi_{\alpha} \omega\right|^{2}+\left(\sum_{i=1}^{p} \mu_{i}\right)|\omega|^{2}=\sum_{\alpha}\left(\lambda_{\alpha}+\frac{\sum_{i=1}^{p} \mu_{i}}{p(n-p)}\right)\left|\Xi_{\alpha} \omega\right|^{2} .
$$

The proof can now be completed as in Proposition 3.4.

This principle can also be applied to $(0,2)$-tensors.

Proposition 3.6. Let $T \in \operatorname{Sym}^{2}(V)$ with $\operatorname{tr}(T)=0$, let $S=\nabla U$ and set

$$
H=\frac{S}{n}-\frac{\operatorname{tr}(S)}{2 n^{2}} I
$$

Let $\lambda_{1} \leq \cdots \leq \lambda_{\left(\begin{array}{c}n \\ 2\end{array}\right)}$ denote the eigenvalues of the weighted curvature tensor $\mathrm{Rm}+h \otimes g$ and suppose that

$$
\lambda_{1}+\cdots+\lambda_{\left\lfloor\frac{n}{2}\right\rfloor} \geq 0 .
$$

If $T$ is $U$-harmonic and $|T|$ has a maximum, then $T$ is parallel. If in addition the inequality is strict, then $T$ vanishes.

Proof. Proposition 3.2(a) implies that

$$
\begin{aligned}
g\left(\operatorname{Ric}_{h \otimes g}(T), T\right) & =-n g\left(\left(H+\frac{\operatorname{tr}(h)}{n} I\right) T, T\right) \\
& =-n g\left(\left(\frac{S}{n}-\frac{\operatorname{tr}(S)}{2 n^{2}} I+\frac{\operatorname{tr}(S)}{2 n^{2}} I\right) T, T\right)=-g(S T, T) .
\end{aligned}
$$


It follows from Proposition 2.1(b) that

$$
\left(\nabla_{X} \nabla_{U}^{*} T\right)(X)+\left(\nabla_{U}^{*} d^{\nabla} T\right)(X, X)=\left(\nabla_{U}^{*} \nabla T\right)(X, X)+\frac{1}{2}\left(\operatorname{Ric}_{\mathrm{Rm}+h \otimes g} T\right)(X, X) .
$$

As in [6, Lemma 2.1 and Proposition 2.9] we conclude that $\operatorname{Ric}_{\mathrm{Rm}+h \otimes g}(T) \geq 0$. When the inequality is strict, the argument shows moreover $\operatorname{Ric}_{\mathrm{Rm}+h \otimes g}(T)>0$ unless $T=0$. This uses again that $T$ is trace-less.

An application of Lemma 2.5 as before implies the claim.

\section{Acknowledgements}

We would like to thank the referees for useful comments.

\section{References}

[1] Bakry D., Émery M., Diffusions hypercontractives, in Séminaire de probabilités, XIX, 1983/84, Lecture Notes in Math., Vol. 1123, Springer, Berlin, 1985, 177-206.

[2] Bakry D., Qian Z., Volume comparison theorems without Jacobi fields, in Current Trends in Potential Theory, Theta Ser. Adv. Math., Vol. 4, Theta, Bucharest, 2005, 115-122.

[3] Lichnerowicz A., Variétés riemanniennes à tenseur C non négatif, C. R. Acad. Sci. Paris Sér. A-B 271 (1970), A650-A653.

[4] Lott J., Some geometric properties of the Bakry-Émery-Ricci tensor, Comment. Math. Helv. 78 (2003), 865-883, arXiv:math.DG/0211065.

[5] Petersen P., Riemannian geometry, 3rd ed., Graduate Texts in Mathematics, Vol. 171, Springer, Cham, 2016.

[6] Petersen P., Wink M., New curvature conditions for the Bochner technique, arXiv:1908.09958v3.

[7] Qian Z., Estimates for weighted volumes and applications, Quart. J. Math. Oxford 48 (1997), 235-242.

[8] Wei G., Wylie W., Comparison geometry for the Bakry-Émery Ricci tensor, J. Differential Geom. 83 (2009), 377-405, arXiv:0706.1120. 